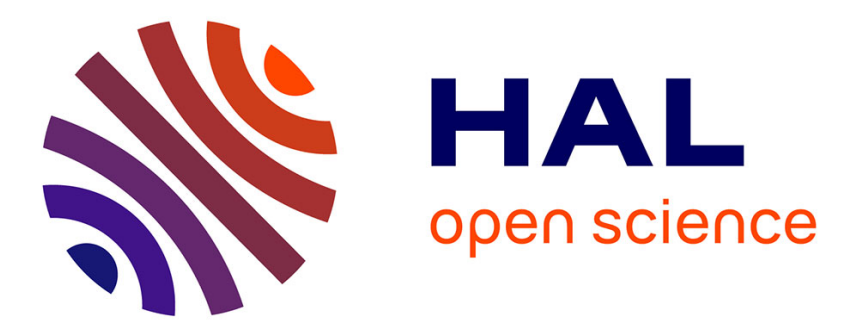

\title{
Current flow and flux pinning properties of YBCO foam struts
}

Michael Rudolf Koblischka, Anjela Koblischka-Veneva, Kévin Berger, Quentin Nouailhetas, Bruno Douine, E. Sudhakar S Reddy, Georg J. J Schmitz

\section{- To cite this version:}

Michael Rudolf Koblischka, Anjela Koblischka-Veneva, Kévin Berger, Quentin Nouailhetas, Bruno Douine, et al.. Current flow and flux pinning properties of YBCO foam struts. IEEE Transactions on Applied Superconductivity, 2019, 29 (5), pp.1-5. 10.1109/TASC.2019.2894712 • hal-01987980

\section{HAL Id: hal-01987980 \\ https://hal.science/hal-01987980}

Submitted on 29 Jan 2019

HAL is a multi-disciplinary open access archive for the deposit and dissemination of scientific research documents, whether they are published or not. The documents may come from teaching and research institutions in France or abroad, or from public or private research centers.
L'archive ouverte pluridisciplinaire HAL, est destinée au dépôt et à la diffusion de documents scientifiques de niveau recherche, publiés ou non, émanant des établissements d'enseignement et de recherche français ou étrangers, des laboratoires publics ou privés. 


\title{
Current flow and flux pinning properties of YBCO foam struts
}

\author{
M. R. Koblischka, A. Koblischka-Veneva, K. Berger, Q. Nouailhetas, B. Douine, E.S. Reddy, and G. J. Schmitz
}

\begin{abstract}
The current flow and the flux pinning properties on struts of superconducting $\mathrm{YBa}_{2} \mathrm{Cu}_{3} \mathrm{O}_{x}$ (YBCO) foams are analyzed in detail in the temperature range $60 \mathrm{~K} \leq T \leq T_{c}$. For this purpose, magnetization loops were measured on foam struts taken from various positions of a $5 \times 2 \times 2 \mathrm{~cm}^{3}$ large foam sample prepared at RWTH Aachen. From these data, the critical current densities, $j_{c}$, and the flux pinning forces, $F_{p}=j_{c} \times B$, were calculated and pinning force scaling diagrams $F_{p} / F_{p \text {,max }}$ vs. $h=H_{a} / H_{\text {irr }}$ were established. The scaling in the temperature range $60 \mathrm{~K}<T<90 \mathrm{~K}$ was found to be well developed for all samples with peak positions, $h_{0}$, above 0.4 , which is an indication of $\delta T_{c}$-pinning. The shape of the pinning functions is found to be completely different from all other high- $T_{c}$ materials and varies only slightly with position. This specific dome-shape cannot be described by an addition of several pinning functions, and the parameters $p$ and $q$ do not fit to the description of Dew-Hughes. Therefore, we employ Kramer plots to obtain more information on the flux pinning mechanism.
\end{abstract}

Index Terms-Foam, YBCO, critical currents, flux pinning, scaling.

\section{INTRODUCTION}

$\mathbf{S}$ UPERCONDUCTING foams are an interesting class of high- $T_{c}$ materials for various applications due to several advantages as compared to conventional bulk samples [1][5]. These advantages include facile oxygenation and effective cooling processes, scalability, light weight of the samples, easy shaping and reduced material costs [6], [7]. The superconducting foams may be useful for a variety of applications, starting from fault-current limiters to trapped field magnets and elements in electric motors and generators, where the reduced weight may play an important role. However, the high$T_{c}$ superconducting foam samples prepared up to now pose many new questions concerning the current flow in such a 3D material and the corresponding flux pinning properties, which are essential to be answered for future applications of these materials. One important issue is the detailed understanding of

Manuscript received xx.xx.xxxx. This work is a part of the SUPERFOAM international project funded by ANR and DFG under the references ANR-17CE05-0030 and DFG-ANR Ko2323-10.

M. R. Koblischka and A. Koblischka-Veneva are with Experimental Physics, Saarland University, P. O. Box 151150, 66041 Saarbrücken, Germany. Present address: Department of Materials Science and Engineering, Shibaura Institute of Technology, 3-7-5 Toyosu, Koto-ku, Tokyo 135-8548, Japan.

K. Berger, and B. Douine are with GREEN, Université de Lorraine, Vandoeuvre-lès-Nancy CEDEX, France.

Q. Nouailhetas is with GREEN, Université de Lorraine, Vandoeuvre-lèsNancy CEDEX, France, and Saarland University, P. O. Box 151150, 66041 Saarbrücken, Germany.

E. S. Reddy is with Raychem RPG Pvt Ltd., Namdev Garnet, Aga Abbas Ali Road Off Halasur Road, Bangalore 560042, India.

G. J. Schmitz is with ACCESS, Intzestrasse 5, 52072 Aachen, Germany. the variation of the microstructure and of the linked superconducting properties throughout the foam structure, which was not addressed in previous works [8]-[10]. This information is a valuable input for modelling of the foam structure, which can make use of attempts already existing in the literature on open-cell polyurethane and metal foams [11], [12].

As mentioned before, the foam samples may be applied as trapped field (TF) magnets, where the improved oxygenation and the reduced weight may enable their use even in space applications. To properly design such systems, modelling of the real foam structure is an important issue, considering both superconducting and mechanical parameters. Therefore, we investigate here in detail the flux pinning properties of in total seven individual foam struts, broken off from one side of the original big foam sample. The study of single foam struts ensures that the current flow is not influenced by percolation effects around the pores. For analysis of the magnetization data, we apply the pinning force scaling approach of DewHughes (DH) [13] to determine the peak positions, $h_{0}$, and the scaling parameters $p$ and $q$, which provide information about the active flux pinning mechanism [14]. As the all foam samples investigated here were found to exhibit a welldeveloped scaling behavior, but the shape of the pinning curves is entirely different from other high- $T_{c}$ superconductors (see, e.g., [15]-[17]), we perform further discussion on the flux pinning properties of the foam struts using Kramer plots [18] following the approach of Oh et al. [19], [20]. The advantage of the Kramer plots to discuss the flux pinning properties is the fact that there are no uncertainities like the determination of the irreversibility fields involved [21].

\section{EXPERIMENTAL PROCEDURE}

Open-cell superconducting foams were produced originally at RWTH Aachen (Germany) on the base of polyurethane foams, which were converted in a first step to $\mathrm{Y}_{2} \mathrm{BaCuO}_{5}$ ("211") foams, and then to $\mathrm{YBa}_{2} \mathrm{Cu}_{3} \mathrm{O}_{7}$ (YBCO or "123") using an infiltration growth (IG) process [22], [23]. More details of the preparation of the foam samples can be found in Refs. [1]-[4]. For the present study, we have employed such a YBCO foam of dimensions $5 \times 2 \times 2 \mathrm{~cm}^{3}$ with a porosity of 40 PPI. Single foam struts named (a) to (g) were broken off the big foam sample on one edge as illustrated in Fig. 1. The thickness of the struts varies between 50 and $100 \mu \mathrm{m}$, while the dimensions in $a$ and $b$ directions range between 0.8 and $1.6 \mathrm{~mm}$. Piece $(\mathrm{g})$ was selected additionally to the other ones as this strut showed a greenish color, which was not seen in the other cases. All struts have the shape of a parallelepiped, 


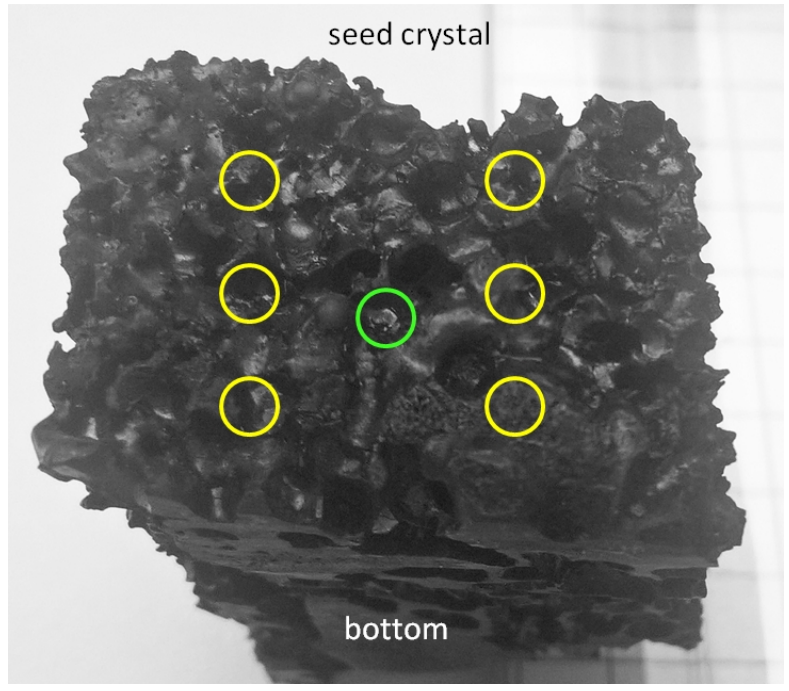

Fig. 1. Photograph of the foam sample with the positions indicated using yellow circles where the foam struts named (a) to (f) were broken out. The first column are samples (a), (c) and (e), from top to bottom, and the second row samples (b), (d) and (f). Sample (g) is marked with a green circle, indicating its greenish appearance.

but both ends are irregularly shaped due to the breaking out of the struts from the big foam piece.

Magnetic measurements were performed using a Quantum Design MPMS3 SQUID system with $\pm 7 \mathrm{~T}$ applied field. In all cases, the field was applied perpendicular to the flat surface of the struts. The field sweep rate was in all cases 0.36 T/min. $M(T)$-diagrams at $1 \mathrm{mT}$ applied field were measured in $\mathrm{FC} / \mathrm{ZFC}$ conditions to determine the superconducting transition temperature, $T_{c}$, and the transition width, $\Delta T_{c}$. These data are summarized in Table I, together with the sample size, the current density obtained at $1 \mathrm{~T}$ applied magnetic field and the scaling parameters $A, p$ and $q$. From the magnetization data, the critical current densities, $j_{c}$, were evaluated using the extended Bean approach [24]. The irreversibility fields, $H_{\text {irr }}$, were determined from the closing of the magnetization loops. As already mentioned in Ref. [25], this closing of the loops is hardly accessible already at $77 \mathrm{~K}$, so all data at lower temperatures were obtained from extrapolations and adjusted accordingly by the DH scaling.

\section{RESULTS AND DISCUSSION}

Figure 2 presents a double-log plot of $j_{c}$ as function of the applied magnetic field, obtained on foam strut (a). The $j_{c}(H)$ curves can be fitted using a power law, $H^{-\alpha}$ with $\alpha$ ranging between $\approx 0.52(60 \mathrm{~K})$ and $0.8(85 \mathrm{~K})$. These data are simillar to ones obtained on coated conductors [26], indicating dominant weak collective flux pinning. Bending down of the curves at high $H$ and $T$ is due to thermal activation.

The overall $j_{c}$-values are always smaller than those of solid, bulk YBCO samples [25]. This observation may be ascribed to the microstructure of the foam struts which differs in two ways from the bulks: (i) The growth sectors induced by the seed crystal are similar to the bulk sample, but the struts are broken off from a 3D system, so the texture is not in (001)-direction, but about $\pm 30^{\circ}$ off [9]. (ii) The struts show the presence of an

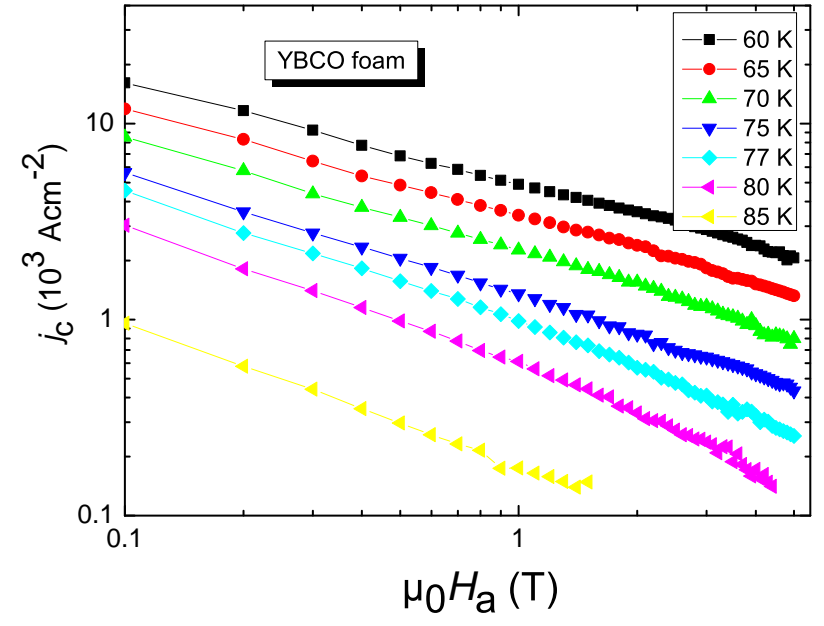

Fig. 2. Critical current density, $j_{c}(H)$ (log-log-plot) measured on foam strut (a).

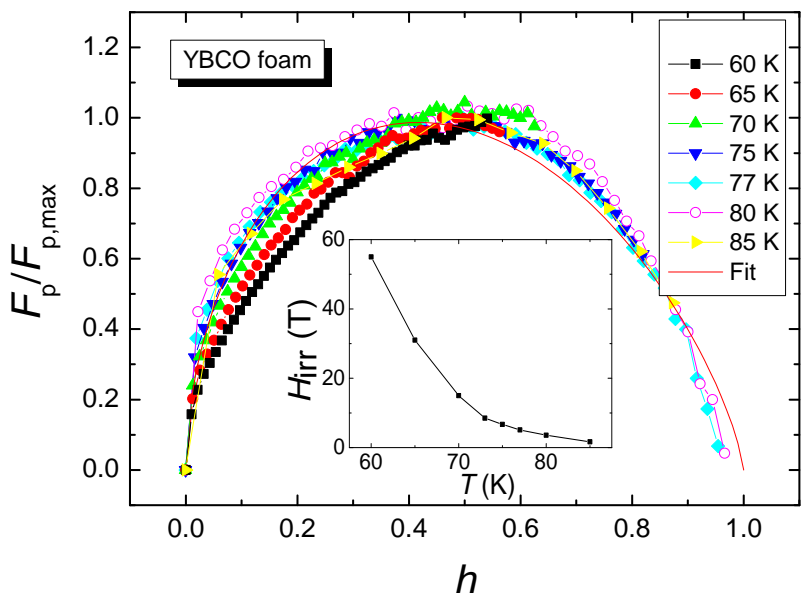

Fig. 3. Pinning force scaling, $f=F_{p} / F_{p, \max }$ vs. $h=H_{a} / H_{\mathrm{irr}}$ of foam strut (a). The red line presents the fit function to all experimental data shown. The inset gives the irreversibility line, $H_{\mathrm{irr}}(T)$, as determined from the closing of the hysteresis loops and adjusted by the scaling.

internal granularity due to embedded grains (YBCO and 211) in the main YBCO matrix and subgrains, which also force the currents within a single strut to flow across grain boundaries (GBs). As the GBs of YBCO are obstacles to the current flow [27], [28] and the struts only have a limited extension, this reduces $j_{c}$ considerably. Table I lists the current densities of all foam struts obtained at an applied field of $1 \mathrm{~T}$, which is already very large for currents across GBs. As consequence, the $j_{c^{-}}$ values are reduced to $700 \ldots 1600 \mathrm{Acm}^{-2}$. The corresponding scaling of the flux pinning forces of sample (a) using the DH scaling is presented in Fig. 3. The scaling of the data is reasonably well developed, and the red line indicates the fit function to all data of this sample. The parameters $p$ and $q$ are determined to be 0.531 and 0.745 , respectively. The parameter $A$ is not a real fit parameter, but defined via the condition $F_{p} / F_{p, \max }\left(h_{0}\right)=1$. The resulting peak position is determined to 0.42 , which is clearly higher than the point pinning with $h_{0}=0.33$, and points therefore, to a contribution of $\delta T_{c^{-}}$ pinning in the foam strut. The inset to Fig. 3 presents the 
TABLE I

PARAMETERS OF THE FOAM STRUT SAMPLES (A)-(G) DETERMINED FROM $M(T)$, AND $M(H)$ MEASUREMENTS AND THE PINNING FORCE SCALING.

\begin{tabular}{|c||c|c|c|c|c|c|c|c|c|}
\hline sample & $\begin{array}{c}T_{c} \\
{[\mathrm{~K}]}\end{array}$ & $\begin{array}{c}\Delta T_{c} \\
{[\mathrm{~K}]}\end{array}$ & $\begin{array}{c}\text { sample size } \\
{\left[\mathrm{mm}^{3}\right]}\end{array}$ & $\begin{array}{c}j_{c}(77 \mathrm{~K}, 1 \mathrm{~T}) \\
{\left[\mathrm{kA} / \mathrm{cm}^{2}\right]}\end{array}$ & $\begin{array}{c}H_{\mathrm{irr}}(77 K) \\
{[\mathrm{T}]}\end{array}$ & $A$ & $p$ & $q$ & $h_{0}$ \\
\hline \hline (a) & 91.1 & 3.2 & $1.5 \times 1.0 \times 0.1$ & 1.2 & 5.7 & 2.347 & 0.531 & 0.745 & 0.42 \\
\hline (b) & 91.2 & 3.5 & $1.3 \times 1.0 \times 0.08$ & 1.5 & 5.8 & 2.252 & 0.502 & 0.689 & 0.44 \\
\hline (c) & 90.7 & 2.7 & $1.6 \times 1.4 \times 0.05$ & 1.4 & 5.5 & 2.107 & 0.497 & 0.583 & 0.46 \\
\hline (d) & 91.2 & 2.9 & $0.8 \times 0.8 \times 0.1$ & 1.3 & 5.7 & 2.235 & 0.526 & 0.643 & 0.45 \\
\hline (e) & 91.0 & 3.5 & $1.1 \times 1.1 \times 0.08$ & 1.6 & 5.7 & 1.99 & 0.477 & 0.517 & 0.48 \\
\hline (f) & 91.1 & 3.3 & $1.4 \times 1.2 \times 0.1$ & 1.6 & 5.8 & 1.893 & 0.434 & 0.489 & 0.47 \\
\hline (g) & 89.7 & 1.5 & $1.2 \times 1.0 \times 0.07$ & 0.7 & 3.7 & 45.6 & 2 & 4 & 0.33 \\
\hline
\end{tabular}
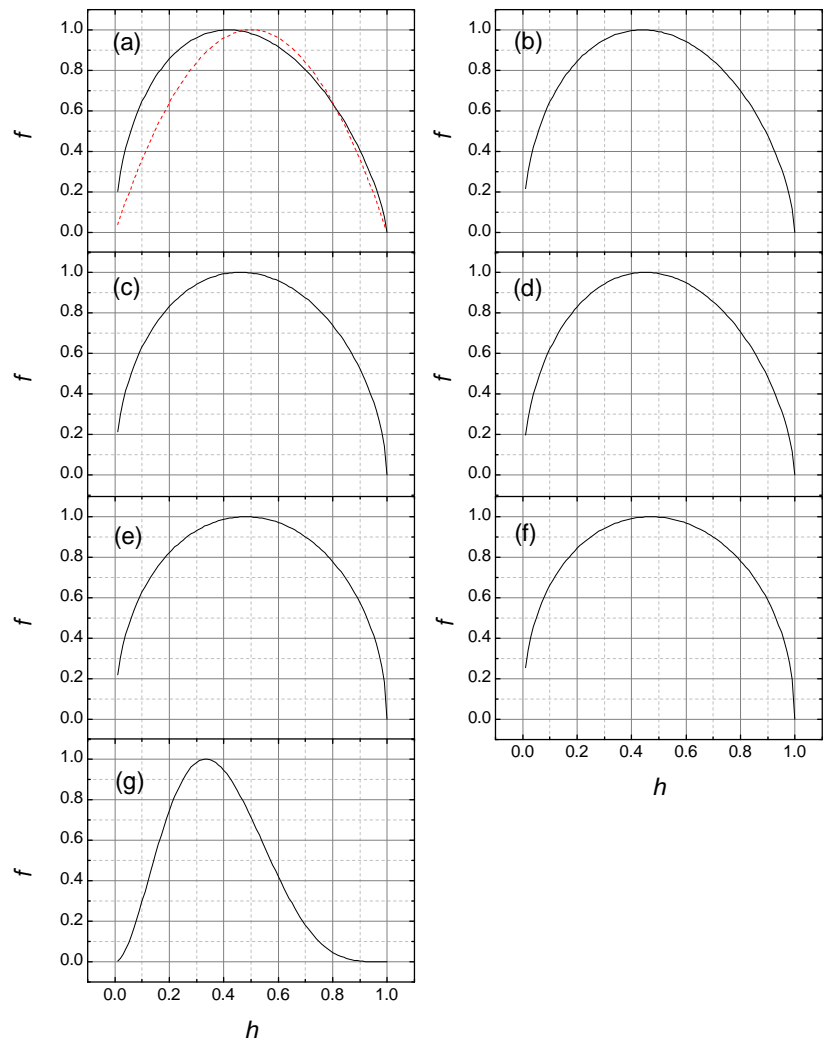

$+$
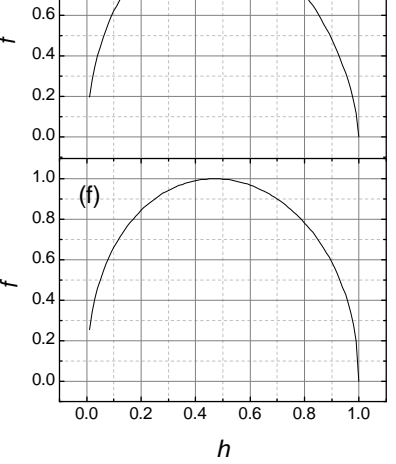

$h$

Fig. 4. Variation of the pinning force scaling $f$ vs. $h$ with position, samples (a)-(g). In all cases, the final fit to all scaling data is shown. The peak positions, $h_{0}$, are found to vary between 0.45 and 0.5 , with the exception of strut $(\mathrm{g})$, where a peak position of $h_{0}=0.33$ is obtained. For the peak positions and the scaling parameters, see Table I. The dashed red line in panel (a) is the DH pinning function with $p=q=1$.

data for $H_{\mathrm{irr}}(T)$, used for the $F_{p}$ scaling. Here it is important to point out that the foam samples share this behavior with the melt-textured, IG processed YBCO bulks as discussed in Ref. [10]. This demonstrates that the specific microstructure with embedded, tiny 211 particles which both materials have in common, is responsible for this peak position and the resulting shape of the pinning function. Table I and Fig. 4 demonstrate that the peak position, $h_{0}$, of the pinning force scaling increases when taking the struts from a larger distance from the seed crystal. Also the values of $j_{c}(1 \mathrm{~T})$ are found to increase in the same manner, whereas $T_{c}, H_{\text {irr }}$ and $\Delta T_{c}$ are not affected by the position in the original foam. This gives a hint that there must be a change in the microstructure of the samples (not in the superconducting material itself), as the oxygen content stays the same indicated by $T_{c}$ and $\Delta T_{c}$ (see Table I). A possible reason for this behavior could be the positive influence of the liquid source on the bottom part of the foam sample in the infiltration processing step, which is placed below the sample. As consequence, the local texture and conversion from the 211 phase may be better developed closer to the liquid source. This is another important issue for future development of the foam samples, which may lead to an optimization of the liquid source arrangement and of the sample shape itself.

Foam strut $(\mathrm{g})$ was selected due its greenish appearance. The pinning force scaling of this strut yields a totally different behavior which resembles that of a standard YBCO sample with a peak position at $h=0.33$. A closer inspection of this sample using SEM-EDX and EBSD revealed that $(\mathrm{g})$ consists of some small YBCO grains embedded in a 211 matrix, i.e., just the opposite of what is expected. The corresponding magnetization and critical current densities measured on sample $(\mathrm{g})$ are consequently smaller than all other struts analyzed; furthermore, the pinning force scaling corresponds to that of a standard YBCO sample. This shows that the specific foam-type microstructure with the numerous GBs and the embedded tiny 211 particles is not realized here. This observation demonstrates that the preparation steps of the large foam samples can still be further optimized to ensure a $100 \%$ conversion into the 123 phase. The peak positions obtained from the $F_{p}$-scaling are for all struts (a)-(f) just below $h_{0}=0.5$, whereas pure YBCO showed a peak position of $\sim 0.33$ indicating a dominant flux pinning provided by nonsuperconducting point defects ( $\delta l$-pinning). The result of this is $h_{0} \approx 0.5$, which is very high for pure YBCO-123 samples [14]. A peak position close to 0.5 can only be provided by additional $\delta T_{c}$-pinning. The consequence from this being that the parameters $p$ and $q$ have in our case values of 0.5 to 0.7 (with $p<q$ always), which is completely different from any pinning function described by $\mathrm{DH}$. In the $\mathrm{DH}$ approach, the parameter $q$ describes the type of pinning, and has hence, only the values 1 and 2 . The parameter $p$ can range between 0 and 2 and gives information about the extension of the flux pinning sites. Therefore, a value of 0.5 for $p$ could be considered as normal, but for $q$ the situation is different. Another point to be discussed here is the shape of the $F_{p}$-scaling curves, which is 


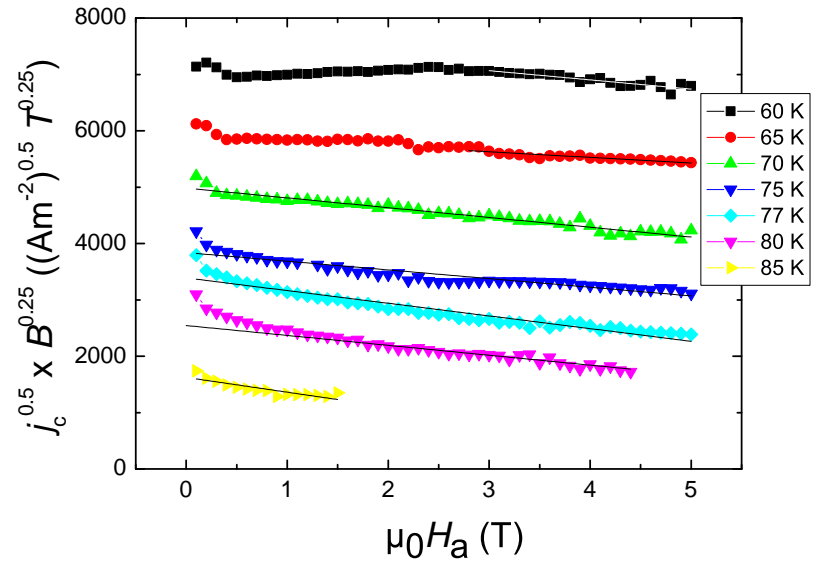

Fig. 5. Kramer plot, $j_{c}^{0.5} \times B^{0.25}$, of the data obtained from foam strut (a).

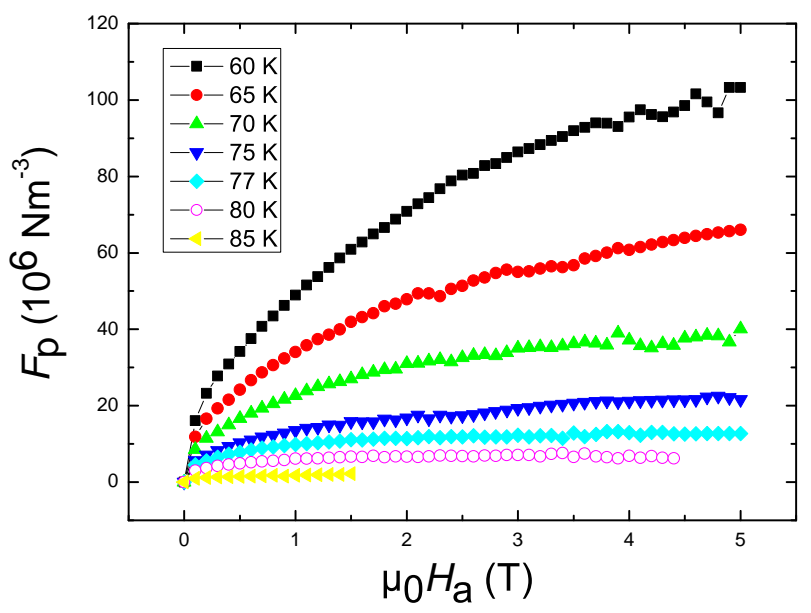

Fig. 6. Dependence of the flux pinning forces, $j_{c} \times B$, on the applied magnetic field of foam strut (a).

completely different from all high- $T_{c}$ superconductors studied in the literature (see, e.g., [15]-[17]). In stark contrast to the commonly reported behavior of RE-123 samples, which gives a quickly rising curve up to $h_{0}$ and then followed by a long tail towards $h=1$, the curves measured on the foam struts have a dome shape. This shape of the pinning force curves is only possible when $p \approx q$, which is similar to the $\mathrm{DH}$ pinning function with $p=q=1$ (see Fig. 4 (a)), describing pinning provided by superconducting (extended) volume pins. However, this function does not properly describe the shape of the present scaling curves at low $h$, which would point to the activity of another pinning mechanism of the $\delta l$-type (surface pins). The data displayed on the low $h$ side in Fig. 3 could be interpreted that the deviations from the fit curve represent a decrease of this surface pinning mechanism when lowering the temperature. Therefore, more information is required to fully understand the properties of the foam samples as well as of the IG-processed YBCO bulks.

Recently, Kramer plots were empolyed to describe the flux pinning properties in $\mathrm{Nb}_{3} \mathrm{Sn}$ [19], [21] and YBCO thin films [20]. In the latter case, the Kramer model including thermal activation was applied. The results obtained there also influ- enced the discussion of the flux pinning properties presented in Ref. [29] concerning pnictide superconductors, where vortex shearing was also considered. The vortex shearing provides the same functional denpendence as the surface $(\delta l)$ pinning. In the Kramer model [18], the pinning force, $j_{c} \times B$, is proportional to $b^{2}(1-b)^{2}$, where the reduced field $b$ is defined as $b=B / B_{c 2}$. The field dependence of $j_{c}^{0.5} \times B^{0.25}$ is, therefore, expected to be a linear function of $B$. This was clearly not fulfilled in the case of YBCO thin films investigated in [20], as the curves showed a strong upturn towards low fields and a linear region can hardly be identified. In Ref. [20], the Kramer model was extended to include thermal activation effects, which could describe then successfully the shape of the curves in the Kramer plots. Remarkably, another recent paper [21] could describe the superconducting property variations in $\mathrm{Nb}_{3} \mathrm{Sn}$ using the GLAG theory, and they could calculate the respective variations of the pinning force scaling and the Kramer plots. This approach could be an interesting step forward also to understand the influence of the changes of the superconducting properties with position in the high- $T_{c}$ superconductors.

Figure 5 presents the Kramer plot of foam strut (a). Our data show a large linear region, and in the low-field region below $0.5 \mathrm{~T}$, an upturn with decreasing $B$ can be observed, which is getting stronger with increasing temperature closer to $T_{c}$. However, we have to note here that for the foam samples, we are mostly below $F_{p, \max }$ in contrast to the situation presented in Ref. [20], where the full range of the pinning curve could be measured. This is illustrated in Fig. 6, where $F_{p}$ is shown as a function of the applied field. Using linear fits to the Kramer function gives an estimate for $B_{c 2}$, which ranges in our case between $6.2 \mathrm{~T}(85 \mathrm{~K})$ and $83 \mathrm{~T}(65 \mathrm{~K})$. These values are clearly much higher than the irreversibility fields determined for the $F_{p}$-scaling as shown in the inset to Fig. 3. Calculating Kramer plots using the approach of [20] leads to a complete description of all our data, which indicates that vortex shearing is important to be considered to describe the flux pinning properties of the superconducting YBCO foam samples.

\section{CONCLusion}

To conclude, we have measured the flux pinning force scaling on seven struts of a superconducting YBCO foam. The DH scaling yields pinning functions with a completely different shape as compared to other high- $T_{c}$ superconductors, and the peak positions range between 0.42 and 0.48 , indicating pinning by the $\delta T_{c}$-type. The current densities and the peak positions of the DH scaling are found to increase when taking struts from positions farer away from the seed, but the shape of the pinning functions and the irreversibility fields remain unchanged. We explain this observation with a change of local microstructure due to the influence of the liquid in the IGprocessing. The Kramer plots show a nearly linear behavior as expected in the Kramer model, which points to the vortex shearing mechanism playing a role.

\section{ACKNOWLEDGMENT}

We acknowledge valuable discussions with S. Pavan Kumar Naik, M. Muralidhar, M. Murakami (Shibaura Institute of Technology, Tokyo, Japan). 


\section{REFERENCES}

[1] E. S. Reddy, and G. J. Schmitz, "Superconducting foams". Supercond. Sci. Technol., vol. 15, 2002, pp. L21-L24.

[2] E. S. Reddy, and G. J. Schmitz, "Ceramic foams". Am Ceram. Soc. Bull. vol. 81, 2002, pp. 35-37.

[3] J. G. Noudem, E. S. Reddy, and G. J. Schmitz, "Magnetic and transport properties of $\mathrm{YBa}_{2} \mathrm{Cu}_{3} \mathrm{O}_{y}$ superconductor foams". Physica C, vol. 390, 2002, pp. 286-290.

[4] E. S. Reddy, M. Herweg, and G. J. Schmitz, "Processing of $\mathrm{Y}_{2} \mathrm{BaCuO}_{5}$ foams". Supercond. Sci. Technol., vol. 16, 2003, pp. 608-612.

[5] J. G. Noudem, E. Guilmeau, D. Chateigner, S. Lambert, E. S. Reddy, B. Ouladdiaf, and G. J. Schmitz, "Properties of $\mathrm{YBa}_{2} \mathrm{Cu}_{3} \mathrm{O}_{y}$-textured superconductor foams". Physica C, vol. 408-410, 2003, pp. 655-656.

[6] J. G. Noudem, "Development of shaping textured $\mathrm{YBaCuO}$ superconductors". J. Supercond., vol. 24, 2011, pp. 105-110.

[7] Ch. Hill, and J. Eastoe, "Foams: From nature to industry". Advances in Colloid and Interface Science, vol. 247, 2017, pp. 496-513.

[8] E. Bartolomé, X. Granados, T. Puig, X. Obradors, E. S. Reddy, and G. J. Schmitz, "Critical state in superconducting single-crystalline $\mathrm{YBa}_{2} \mathrm{Cu}_{3} \mathrm{O}_{7}$ foams: Local versus long-range currents". Phys. Rev. B, vol. 70, 2004, Art. no. 144514.

[9] M. R. Koblischka, A. Koblischka-Veneva, E. S. Reddy, and G. J. Schmitz, "Analysis of the microstructure of superconducting YBCO foams by means of AFM and EBSD”. J. Adv. Ceram., vol. 3, 2014, pp. 317-325.

[10] A. Koblischka-Veneva, M. R. Koblischka, N. Ide, K. Inoue, M. Muralidhar, T. Hauet, and M. Murakami, "Microstructural and magnetic analysis of a superconducting foam and comparison with IG-processed bulk samples". J. Phys. Conf. Ser., vol. 695, 2016, Art. no. 012002.

[11] M. D. Montminy, A. R. Tannenbaum, and C. W. Macosko, The 3D structure of real polymer foams. J. Colloid Interface Sci., vol. 280, 2004, pp. 202-211.

[12] Zh. Nie, Y. Lin and Q. Tong, Modeling structures of open cell foams. Comput. Mater. Sci., vol. 131, 2017, pp. 160-169.

[13] D. Dew-Hughes, "Flux pinning mechanisms in type-II superconductors". Phil. Mag., vol. 30, 1974, pp. 293-305.

[14] M. R. Koblischka, and M. Murakami, "Pinning mechanisms in bulk high- $T_{c}$ superconductors". Supercond. Sci. Technol., vol. 13, 2000, pp. 738-744.

[15] M. R. Koblischka, "Pinning forces and scaling in high- $T_{c}$ superconductors". Physica C, vol. 282-287, 1997, pp. 2193-2194.

[16] V. Sandu, "Pinning force scaling and its limitation in intermediate and high-temperature superconductors". Mod. Phys. Lett. B, vol. 26, 2012, Art. no. 1230007.
[17] M. R. Koblischka, and M. Muralidhar, "Pinning force scaling analysis in Fe-based high- $T_{c}$ superconductors". Int. J. Mod. Phys. B, vol. 30, 2017 , Art. no. 1630017.

[18] E. J. Kramer, "Scaling laws for flux pinning in hard superconductors". J. Appl. Phys., vol. 44, 1973, pp. 1360-1370.

[19] S. Oh, and K. Kim, "A consistent description of scaling laws for flux pinning in $\mathrm{Nb}_{3} \mathrm{Sn}$ strands based on the Kramer model". IEEE Trans. Appl. Supercond., vol. 17, 2007, pp. 3898-3900.

[20] S. Oh, H. Choi, Ch. Lee, S. Lee, J. Yoo, D. Youm, H. Yamada, and $\mathrm{H}$. Yamasaki, "Relation between the critical current and the $n$ value of REBCO thin films: A scaling law for flux pinning of REBCO thin films". J. Appl. Phys., vol. 102, 2007, Art. no. 043904.

[21] Y. Li, and Y. Gao, "GLAG theory for superconducting property variations with A15 composition in $\mathrm{Nb}_{3}$ Sn wires". Sci. Rep., vol. 7, 2017, Art. no. 1133.

[22] K. Iida, N. H. Babu, Y. Shi, and D. A. Cardwell, "Seeded infiltration and growth of large, single domain $\mathrm{Y}-\mathrm{Ba}-\mathrm{Cu}-\mathrm{O}$ bulk superconductors with very high critical current densities". Supercond. Sci. Technol., vol. 18, 2005, pp. 1421-1427.

[23] N. D. Kumar, T. Rajasekharan, K. Muraleedharan, A. Banerjee, and V. Seshubai, "Unprecedented current density to high fields in $\mathrm{YBa}_{2} \mathrm{Cu}_{3} \mathrm{O}_{7 \delta}$ superconductor through nano-defects generated by preform optimization in infiltration growth process", Supercond. Sci. Technol., vol. 23, 2010, Art. no. 105020 .

[24] D. X. Chen, and R. B. Goldfarb, "Kim model for the magnetization of hard superconductors". J. Appl. Phys., vol. 66, 1989, pp. 2489-2500.

[25] M. R. Koblischka, A. Koblischka-Veneva, C. Chang, T. Hauet,E. S. Reddy, and G. J. Schmitz, "Flux pinning analysis of superconducting YBCO foam struts". IEEE Trans. Appl. Supercond., accepted for publication.

[26] Ö. Polat, J. W. Sinclair, Y. L. Zuev, J. R. Thompson, D. K. Christen, S. W. Cook, D. Kumar, Y. Chen, and V. Selvamanickam, "Thickness dependence of magnetic relaxation and $\mathrm{E}-\mathrm{J}$ characteristics in superconducting Gd-Y)-Ba-Cu-O films with strong vortex pinning”. Phys. Rev. B, vol. 84 2011, Art. no. 024519.

[27] D. C. Larbalestier, A. Gurevich, D. M. Feldmann, and A. Polyanskii, "High- $T_{c}$ superconducting materials for electric power applications", Nature, vol. 414, 2001, pp. 368-377.

[28] J. Ayache, "Grain boundaries in high temperature superconducting ceramics", Phil. Mag., vol. 86, 2006, pp. 2193-2239.

[29] C. Tarantini, K. Iida, J. Hänisch, F. Kurth, J. Jaroszynski, N. Sumiya, M. Chihara, T. Hatano, H. Ikuta, S. Schmidt, P. Seidel, B. Holzapfel, and D. C. Larbalestier, "Intrinsic and extrinsic pinning in $\mathrm{NdFeAs}(\mathrm{O}, \mathrm{F})$ : vortex trapping and lock-in by the layered structure". Sci. Rep., vol. 6, 2016, Art. no. 36047 . 